Revue d'histoire de l'enfance « irrégulière »

Le Temps de l'histoire

$12 \mid 2010$

Autour de l'enfant : la ronde des professionnels

\title{
La police des mineurs à l'heure de la prévention (1935-1966) : une mission oubliée ?
}

Juvenile police when prevention dominated (1935 - 1966): a forgotten role?

\section{Mathias Gardet}

\section{(2) OpenEdition}

Journals

Édition électronique

URL : http://journals.openedition.org/rhei/3189

DOI : 10.4000/rhei.3189

ISBN : 978-2-7535-1651-9

ISSN : $1777-540 \mathrm{X}$

Éditeur

Presses universitaires de Rennes

Édition imprimée

Date de publication : 30 novembre 2010

Pagination : 111-137

ISBN : 978-2-7535-1259-7

ISSN : $1287-2431$

\section{Référence électronique}

Mathias Gardet, «La police des mineurs à l'heure de la prévention (1935-1966) : une mission

oubliée? », Revue d'histoire de l'enfance « irrégulière » [En ligne], 12 | 2010, mis en ligne le 30 novembre

2010, consulté le 30 avril 2019. URL : http://journals.openedition.org/rhei/3189; DOI : 10.4000/

rhei.3189

Ce document a été généré automatiquement le 30 avril 2019

(c) PUR 


\title{
La police des mineurs à l'heure de la prévention (1935-1966) : une mission oubliée?
}

Juvenile police when prevention dominated (1935 - 1966): a forgotten role?

\author{
Mathias Gardet
}

1 Si tout le monde se souvient dans le film La fureur de vivre ${ }^{1}$ de la scène de la bagarre au couteau et de la course de voitures en haut de la falaise, peu de personnes se rappellent de la toute première scène où l'on assiste à la déambulation hasardeuse d'un James Dean complètement ivre avant qu'il ne s'effondre au milieu de la chaussée et qu'il ne soit ramassé par la police pour être emmené au poste. Or, le commissariat où il est transféré est d'un type bien particulier, ainsi que l'indique l'inscription au-dessus de la porte d'entrée que l'on aperçoit au passage: Police station, division 6, juvenile division... La clientèle interrogée par les officiers de police est en effet exclusivement composée de mineurs (un garçon à qui l'on donnerait tout juste 15 ans et une jeune fille incarnée par Nathalie Wood, qui n'a effectivement à l'époque que 17 ans) ; James Dean, bien qu'il ait 24 ans au moment du tournage, est censé lui-même incarner le rôle du jeune Jim Stark, un teen-ager (américanisme dérivé de -teen, finale des nombres de thirteen, treize, à nineteen, dix-neuf), vivant avec ses parents. L'inspecteur chargé de s'entretenir avec l'adolescent se démarque très vite de la pratique de l'interrogatoire pour s'investir dans une relation de proximité avec le jeune à mi-chemin entre une image paternelle et celle du grand-frère, l'invitant à venir le consulter s'il ressent à nouveau son mal-être; une position que l'on s'attendrait plutôt à voir occupée en France par un éducateur à cette époque.

Ce modèle américain d'une police spécialisée dans la prévention de la délinquance juvénile a pourtant fait des émules en France, comme le montre par exemple l'article publié par Hélène Campinchi ${ }^{2}$ sous le titre " La prévention de la délinquance juvénile dans un service de police aux Etats-Unis » dans la revue Rééducation ( ${ }^{\circ} 53$ de janvier-février 1954). Elle évoque le fonctionnement du bureau d'aide à la jeunesse, le "Juvenile Aid Bureau », connu au départ sous le nom de "Bureau de prévention criminelle », créé en 1930 , sous le contrôle direct du $6^{\mathrm{e}}$ directeur adjoint de la police de New York et doté 
d'unités spéciales composées de fonctionnaires de police, hommes et femmes chargés d'un travail de patrouille. Elle insiste en particulier sur l'existence en son sein d'une association sportive, la PAL "Police Athletic League », qui gère 75 centres de jeunesse, ouverts de $15 \mathrm{~h}$ à $22 \mathrm{~h}$, offrant aux jeunes des jeux et sports les plus divers : athlétisme, basket-ball, tennis, boxe, etc. en même temps qu'y sont organisées des séances récréatives de musique, de danse, d'art dramatique. La PAL a également un camp d'été où 500 garçons de 10 à 15 ans font un séjour de 3 semaines par an.

Il en va de même pour la communication intitulée «Les relations de la Police avec l'enfance ", faite par Henri Joubrel ${ }^{3}$ devant le comité de la Fédération internationale des fonctionnaires supérieurs de la police réuni à Lisbonne du 26 au 30 juin 1956, à l'issue d'un voyage aux Etats-Unis. En prenant appui sur l'exemple anglo-saxon, ce dernier propose une série de moyens à mettre en œuvre " pour que le policier ne paraisse plus, aux yeux des jeunes, être surtout l'agent répressif, mais aussi le fonctionnaire protecteur et amical ", espérant ainsi qu'il déclenche admiration et pourquoi pas identification. Il fait neuf suggestions dans un crescendo pour le moins étonnant:

- Que le policier devienne un des agents clés de l'enseignement de la prévention routière en proposant même parfois une coopération des élèves à la police de la circulation automobile à la sortie des écoles.

- Casser le cliché du policier en uniforme en formant des équipes sportives qui permettent, sans établir un contact direct avec les jeunes, de leur donner sur les stades ou dans la presse, l'occasion d'admirer les performances physiques.

- Admettre des jeunes du quartier au sein des associations sportives ou de loisir créées en certains endroits pour les enfants de policier.

- Organiser des fêtes auxquelles seraient conviés les jeunes et dont le bénéfice servirait à une réalisation exemplaire à leur profit (construction d'une piscine par exemple).

- Faire participer des policiers à des équipes sportives de jeunes « en danger moral » dans les quartiers populaires.

- Animer en tant que "moniteurs» des groupes de prévention sociale organisant jeux d'intérieur, prêts de livres et d'illustrés, causeries, ciné-clubs et télé-clubs, chorales, soirées récréatives, séances d'entraînement à l'athlétisme, au judo, au cyclisme, à la natation et au nautisme, sorties de dimanche ou de week-end, cantonnements à la montagne l'hiver, camps d'été.

- Établir des contacts avec les responsables des mouvements de jeunesse ou groupements de jeunesse implantés dans les zones socialement défavorisées (notamment les mouvements scouts).

- Mettre en place des causeries faites par des policiers dans les organisations de jeunes sur les structures, les caractéristiques de la ville, du département, de la région, de l'État et tout ce qui concerne les droits et devoirs du citoyen (remplaçant en quelque sorte l'instruction civique dans les écoles, considérée comme un échec).

- Créer un service « aimable» de renseignements pour les jeunes ayant des questions d'ordre pratique à poser.

4 La condition posée pour la réussite de ces injonctions repose sur une formation particulière de policiers spécialement recrutés et bien rémunérés. Henri Joubrel suggère notamment qu'ils effectuent de véritables stages en tant qu'éducateurs provisoires dans les établissements d'accueil pour jeunes inadaptés ou dans les services dits de «milieu ouvert $»^{4}$. Le plus surprenant des propositions émises par Henri Joubrel, c'est qu'elles ne sont pas restées lettres mortes et que, rejoignant un courant réformiste qui s'étend au 
sein des différents corps de police, elles ont inspiré des expériences pilotes au tournant des années 1950-1960, aujourd'hui totalement oubliées. Elles ont notamment contribué à la naissance de la brigade des mineurs dont les attributions actuelles semblent avoir abandonné une grande partie du champ d'intervention des débuts.

Comment donc est apparu ce nouvel agent qualifié au départ par les professionnels de policier " mineur ", faisant ainsi à la fois allusion à sa clientèle ${ }^{5}$ mais aussi à la difficile affirmation de son identité au sein des corps de police très institués? «Police des mineurs, grande police? " s'interroge à son tour en 1958 Fernand Zamaron, un de ses premiers responsables qui défend avec fougue la nécessité d'en généraliser la création dans toute la France. Prévention et non plus seulement répression martèle sans relâche l'ensemble des revues de la police au cours des années 1950-1960, au point de se demander si ce leitmotiv ne faisait pas figure de méthode Coué pour surmonter la force des traditions et les impensables de la culture policière.

\section{Un service social de la police?}

6 Parmi les premiers auxiliaires spécialisés pour s'occuper des mineurs au sein de la Police, expérimentés en France au milieu des années 1930, figurent des femmes désignées sous l'appellation «d'assistantes de police». Geneviève Pruvost ${ }^{6}$, dans ses travaux sur la difficile féminisation de la police, évoque la mise en place d'un tout premier service de ce type en 1935 à Paris. Le lancement est bien modeste: elles ne sont que deux au démarrage, 4 en 1937, 5 entre 1939 et 1941 alors qu'il y a 20 postes à pourvoir, plus que 2 en 1942, 19 en 1943, 26 en 1944 et 24 en 1949-1950. Audrey Ambraise dans ses recherches sur la ville de Lyon évoque le projet de copier l'initiative parisienne à partir de 1936. Là encore le démarrage de ce service est timide : 2 assistantes de police sont recrutées en 1937, 2 autres postes suivent tardivement en mai 1943 puis en avril 1945, avant d'être renforcées par 7 autres embauches appelées à intervenir dans un rayon d'action plus vaste : Roanne, Montluçon, Vichy et enfin Clermont-Ferrand entre 1945 et 1946 ${ }^{7}$. D'autres expériences similaires ont été menées à Grenoble ${ }^{8}$ et à Marseille notamment, sans qu'une généralisation de ce statut ne réussisse à être adoptée au niveau national.

7 Si cette entrée au compte-goutte des femmes dans les métiers de la police est l'aboutissement laborieux des revendications des mouvements féministes de l'époque en particulier du Conseil national des femmes françaises -, en revanche leur appellation, leur tenue et leur mission, correspondent bien au triomphe et à l'extension de la profession d'assistantes sociales à cette période. En effet, alors que jusque dans les années 1920 le terme même d'« assistante sociale » est bien difficile à cerner, le Conseil supérieur de l'assistante publique ayant même refusé en 1924 d'en reconnaître officiellement l'existence en arguant que le service social ne constituait pas à proprement parler l'exercice d'une profession, dix ans plus tard, elles sont omniprésentes. Leur sphère d'influence s'est étendue à l'hôpital, à l'école, à l'armée, dans les communes, auprès des tribunaux pour enfants et des assurances sociales, etc.; elles ont de plus obtenu du ministère de la Santé publique, tout d'abord un brevet de capacité professionnelle le 12 janvier 1932 leur permettant de porter le titre d'assistante de service social de l'État français, puis le 17 février 1938, un diplôme d'État fusionné avec celui des infirmières (au dépens de ces dernières), qui signe pour de nombreuses années leur prédominance dans le domaine de l'action sociale9. Malgré l'amnésie qui semble avoir frappé la mémoire et 
les histoires du service social ainsi que de la profession d'assistante sociale quant à leur contribution décisive dans la formation des premières assistantes de police, c'est bien dans l'optique de cette concordance que l'on doit décrypter les attributions données à ces dernières.

Bien que la couleur de leur manteau soit de la teinte «bleu préfecture », leur costume «demi-civil», constitué en outre d'un chemisier bleu clair, d'une jupe bleue, d'un chapeau de feutre et de brodequins ressemble à s'y méprendre à l'uniforme de leurs consœurs assistantes sociales. Elles sont pour la plupart recrutées parmi les jeunes filles et femmes âgées de 25 à 40 ans et doivent être pourvues le plus souvent du diplôme d'État d'assistante sociale (une possibilité de dispense étant offerte à celles qui sont déjà infirmières diplômées ou titulaires d'un diplôme d'enseignement supérieur, mais à la condition qu'elles aient déjà deux ans de pratique dans un service social).

9 Si dans l'optique des mouvements féministes qui défendent le projet en 1935, les assistantes de police devaient constituer des brigades qui, à l'égal de leurs collègues masculins, seraient chargées de missions de patrouille et de surveillance, avec une spécialisation sur les questions de prostitution des femmes, elles sont en fait reléguées dans un premier temps dans le domaine de la protection de l'enfance, notamment dans la répression du vagabondage scolaire. Théoriquement, elles peuvent exercer le droit d'arrestation, mais sont interdites du port d'arme. Au début des années 1950, leur présence sur la voie publique devient exceptionnelle, elles abandonnent le «travail de rue " pour se cantonner dans "le travail à domicile». Elles retrouvent ainsi leur spécialité acquise durant leur formation dans les différentes écoles de service social: affectées à un secteur déterminé, elles procèdent à des enquêtes concernant de jeunes enfants qu'elles rédigent dans des bureaux après intervention auprès des familles. Leur "sensibilité et délicatesse féminine " peuvent alors se conjuguer "harmonieusement avec l'autorité masculine » sans faire ombrage ni concurrence à leurs collègues policiers :

"Admettez que si l'on entend frapper à sa porte et qu'à la question : "Qui est là ?" réponde le mot "Police", l'entendre d'une voix masculine ou féminine crée une sensation toute différente. Que dans le ménage ainsi visité ce soit l'homme ou la femme qui endosse une responsabilité, un sentiment de quelque honte mais d'une honte salutaire, honorable si l'on peut dire, va dès l'entrée de l'assistante déjà atteindre le coupable. Il va falloir s'expliquer avec une jeune femme correcte et douce, mais tenace, curieuse en diable, et dont la demande, au-delà des questions posées envisage déjà ce problème : "Comment vais-je obtenir la confiance de ces gens pour qu'ils acceptent mes conseils et mon aide ?" $»^{10}$

10 Si le service des assistantes de police parisien devient actif en étant rattaché en 1942 à la direction de la Police judiciaire et si en principe elles ont dorénavant un statut d'inspecteur (même grade, même traitement, mêmes pouvoirs, mêmes droits), il est cependant précisé que contrairement aux officiers de police, elles ne peuvent accéder aux postes de commissaires-adjoints puis de commissaires ${ }^{11}$. Par ailleurs, bien qu'il soit affirmé qu'elles ne sont pas appelées à "devenir de pures assistantes sociales ${ }^{12}$, les différences avec ces dernières sont de plus en plus ténues d'autant que les besoins des tribunaux pour enfants sont croissants en matière d'enquêtes :

«Oui, je n'ignore pas qu'il n'existe que peu d'assistantes sociales du service de la protection de l'enfance et de l'adolescence en danger près des Parquets ; telle cette agglomération de 220.000 habitants qui n'en dispose que d'une seule. L'on conçoit que les magistrats de ce siège soient contraints par la force des choses d'avoir recours aux policiers de la brigade des mineurs, [...]. Le corps des assistantes sociales ne suffit plus, bon nombre d'entre elles ont tendance à s'orienter vers le 
secteur privé (usines, entreprises...). Il serait nécessaire de créer au ministère de la

Justice un nouveau corps : celui d'enquêteur social. ${ }^{13}$

11 La proximité du profil entre assistantes de police et assistantes sociales est d'autant plus recherchée que les services de protection des mineurs qui se mettent petit à petit en place dans les principales villes cherchent à entretenir des rapports privilégiés avec les services sociaux avoisinants ${ }^{14}$.

\section{Quand les hommes s'en mêlent}

12 Le rattachement des assistantes de police en tant que service actif ${ }^{15}$ de la direction de la Police judiciaire en 1942 est présenté dans la plupart des écrits policiers comme étant à la fois une reconnaissance de l'action menée par ces pionnières et de l'arrivée des hommes dans ce domaine d'intervention, qui du même coup prennent la main. Alors que les assistantes de police avaient obtenu avec difficulté la nomination d'une assistante principale, chef de service, la mise en place du service actif, qui se transforme, le 31 mars 1943, en service spécial de protection des mineurs et de la natalité, installé au 14 puis au 12 quai de Gesvres ( $4^{\mathrm{e}}$ arrondissement) est en effet placé dorénavant sous l'autorité d'un commissaire divisionnaire et d'un commissaire de police. Il se renforce d'une brigade d'inspecteurs (qui au nombre de 26 en 1949 dépasse celle des 24 assistantes de police) et comprend une section "enquêtes ", exécutées soit spontanément soit à la demande des tribunaux pour enfants, une section "disparitions ", une section " des pièces de justice » qui exécute les ordonnances de comparution et les arrestations des mineurs décidées par les tribunaux pour enfants ainsi que les conduites dans les lieux de placement imposés, une section « voie publique » qui recherche les délinquants et les vagabonds et surveille les lieux publics ${ }^{16}$.

13 Cette chronologie demande cependant à être révisée, un rapport d'activité du tribunal pour enfants et adolescents de la Seine daté de 1931 signale en effet dès le mois de juin 1930 l'existence d'une première brigade ou section spéciale de la Protection des mineurs de la police judiciaire qui aurait été créée à Paris à l'initiative de M. Chiappe, préfet de police, dirigé par M. Priolet, commissaire spécial à la direction de la Protection judiciaire et composée de 11 inspecteurs et deux brigadiers ${ }^{17}$. Pourquoi cette première expérience semble avoir été oubliée par la suite? Seule une recherche dans les archives de ces services permettrait d'en découvrir les tenants et aboutissants. Toujours est-il qu'en 1943, dans la répartition des tâches au sein du nouveau service spécial de protection des mineurs et de la natalité, il est clair que l'essentiel des activités sera assuré par les inspecteurs et que celles assignées aux assistantes, le seront sous tutelle masculine. La consolidation de ce service au sein de la Police judiciaire à la fin des années 1940 est suivie d'une série d'initiatives locales presque concomitantes qui montre la diffusion progressive de cette spécialisation dans les différents corps de police masculins français et sa plus grande visibilité.

En 1954, les Cahiers de l'enfance, revue tout juste fondée par le journaliste Alexis Danan, consacre plusieurs articles à l'expérience menée à Nantes, en 1949-1950, par le commissaire principal Bonhomme qui a créé une brigade de deux agents cyclistes uniquement chargée de veiller à la fréquentation scolaire. L'innovation du «système Bonhomme », tel qu'il est présenté, réside essentiellement dans le fait qu'il « décale ainsi le temps de l'intervention policière en devenant préventive $»^{18}$. 
15 En 1957, Louis Casali, éducateur spécialisé et rédacteur en chef de la revue Liaisons, bulletin de l'ANEJI ${ }^{19}$, publie un reportage qu'il a effectué lui-même quelques mois auparavant à Cherbourg, pour rendre compte d'une autre tentative expérimentée en 1951 par le commissaire principal Permey. Ce dernier a institué un service spécialisé chargé de la surveillance et de la protection de l'enfance délinquante confié à un inspecteur, secondé par deux gardiens de la paix. Louis Casali nous entraîne sur les pas de Maxime Leluan, présenté comme "policier éducateur " et faisant fonction "à la fois d'assistant social en procédant à des enquêtes, de conseiller social, d'éducateur en organisant des confrontations entre enfants et parents, de délégué à la liberté surveillée en proposant des séances de culture physique deux fois pas semaine». Outre son aura d'ancien résistant, il est précisé qu'il a été recruté pour ses aptitudes psychologiques et ses connaissances acquises auprès des jeunes (il était entraîneur athlétique depuis 1944 ; son nom sera d'ailleurs donné plus tard à une salle de gymnastique de la ville de Cherbourg). Nous sommes en novembre 1956, un samedi soir, et nous pénétrons dans l'atmosphère enfumée d'un dancing sur le port :

«Les doubles portes s'ouvrent sur la nuit, un couple sort, nous entrons : lumière, musique, parfums, des couples enlacés sur la piste, au comptoir des serveuses assiégées. C'est avec un vague sentiment de gêne que je suis entré à la suite de l'inspecteur Leluan: j'en suis pour mes frais. Même brouhaha, même atmosphère, les rires fusent encore, les couples dansent toujours, et du fond de la salle, émergeant des têtes, une main amicale salue notre présence. "M. Maxime" entre dans son domaine... La table où nous sommes installés se trouve entourée par une bande de jeunes qui ont quitté le bar. "Alors, M. Maxime, comment ça s'est terminé cette partie de ping-pong hier soir? Vous avez gagné je parie ?" Hier soir, l'inspecteur Leluan, dans un club de la ville a battu tous ses adversaires, et tous le savent ici. On plaisante, on parle sport, boulot, loisirs. "Je vous attends demain matin sur le stade, à 9 heures, vous en avez bien besoin les gars, et puis, pour le ping-pong, je vous accorde dix points d'avance au prochain match". "Les gars" ont 22,23 ans. M. Maxime, 51 ans ! $»^{20}$

En 1960, Paul Coupet, commissaire de police, rédige à son tour un article dans la Revue moderne de la police, dans lequel il se réfère à l'impulsion donnée en mars 1952, par le procureur de la République de Toulouse qui aurait demandé et obtenu du commissaire principal, chef de la Sûreté de la ville, de spécialiser deux fonctionnaires de police (un officier et un inspecteur volontaires) dans les questions relevant de l'enfance et de l'adolescence. Devant le succès de l'entreprise, le service ainsi créé s'est renforcé progressivement d'une brigade de 10 inspecteurs donnant naissance à une "évolution nouvelle de la fonction de police » que Paul Coupet baptise "policier mineur » et qui se caractérise par « son sens social » et une action avant tout préventive :

" Dans toutes ses démarches, le policier « mineur » a été amené à adopter à l'égard de l'adolescent ou de sa famille une attitude dépouillée de tout esprit systématiquement répressif mais empreinte de compréhension humaine, réaliste et réfléchie. »

Paul Coupet évoque alors la " révolution lente, peu discernable peut-être, mais certaine » qui s'est produite au sein même du corps de police :

« Le policier "mineur" qui, au début, était souvent l'objet des railleries de certains de ses collègues, est actuellement apprécié par tous ; bien plus - prenant ainsi une inconsciente mais éclatante revanche sur ses premiers détracteurs - il a sans le vouloir imprégné ses collègues des autres brigades de l'esprit nouveau qui anime ses interventions quotidiennes. ${ }^{21}$ 
C'est l'expérience de Toulouse, pourtant pas la première, qui est la plus souvent mentionnée comme étant à l'origine de la brigade des mineurs. Peut-être est-ce dû en partie à sa plus grande visibilité procurée par la thèse de droit de Geneviève Montagne rédigée en $1956^{22}$. D'autres écrits témoignent de la multiplicité de ce type d'entreprises au début des années 1950 sur le territoire national, notamment à Nancy, au Havre, à Lyon, en Seine-et-Marne..., dont le recensement plus précis reste à faire. Malgré la grande publicité qui leur est donnée dans les revues de la Police, il faudrait étudier de près leur fonctionnement effectif sur le terrain et leur durée.

\section{Prévention et spécialisation, un mot d'ordre à l'international}

Cet engouement apparent pour la formule alliant prévention et affectation d'un personnel spécifique pour s'occuper des affaires de mineurs de la part des principaux fonctionnaires de police s'inspire en fait de consignes et de modèles débattus sur la scène internationale par l'intermédiaire d'organismes comme l'Unesco, les Nations Unies, l'Organisation internationale de police criminelle-Interpol (en abrégé OIPC-Interpol ou tout simplement Interpol) ou la toute nouvelle Fédération internationale des fonctionnaires supérieurs de la police et, au niveau national, dans une concertation de plus en plus grande entre les directions des services de police et le ministère de la Justice.

Lors de son assemblée générale d'octobre 1960 qui se déroule à Washington, l'OIPCInterpol fait un rappel de toutes les mesures adoptées sur la question des rapports entre la police et la délinquance juvénile. Tout en se référant aux débats menés dans l'entredeux-guerres, l'organisation insiste en particulier sur le principe de la spécialisation des services de police judiciaire traitant ce genre d'affaires discuté puis adopté lors de la $16^{\mathrm{e}}$ session de juin 1947 à Paris et de la $17^{e}$ session de septembre 1948 à Prague. L'année suivante, lors de l'assemblée générale qui se déroule à Berne, l'accent est mis non plus seulement sur la répression, mais aussi sur la prévention, ce qui amène Interpol à étudier dans ses réunions suivantes le rôle social de la police ${ }^{23}$. En 1955-1956, les numéros 90 et 95 de la Revue internationale de police criminelle (publication officielle de l'organisation) sont consacrés aux cercles ou clubs de jeunes de la police, présentés comme une des meilleures formes de prévention de la délinquance et dont est préconisée la mise en place généralisée. Durant la $25^{\mathrm{e}}$ session de l'assemblée générale de juin 1956 à Vienne, le secrétariat général présente un programme-type de formation des policiers chargés de la police des mineurs, reposant sur 3 principes : spécialisation, volontariat, sélection. Il est ainsi affirmé qu'« au droit pénal nouveau des mineurs doit correspondre une police des mineurs », que «sa spécialisation est indispensable, qu'il s'agisse de sections spécialisées au sein de services généraux ou de services autonomes de mineurs » et que « le personnel ne doit se composer, en principe, que de volontaires ayant reçu (après sélection sévère) une formation spéciale $»^{24}$.

Parallèlement l'Unesco, lors de la conférence sur « les problèmes d'éducation dans leurs rapports avec la santé mentale des enfants en Europe » qui se déroule à Paris le 20 octobre 1952, laisse une large place à l'exposé de la Fédération internationale des fonctionnaires supérieurs de la police. Créée en 1950, cette dernière a été dotée entre temps du statut consultatif officiel auprès de l'Unesco et du Conseil de l'Europe; son secrétaire général est Paul Villetorte, commissaire principal de la Sûreté nationale à 
Paris. Le rapport de quatre pages qu'elle présente, très largement diffusé, fait aussi "prévaloir la prévention sur la répression » et insiste sur la formation professionnelle particulière qu'il conviendrait de donner aux fonctionnaires de police pour réussir à instaurer «un climat de confiance entre l'enfant et la Police». Sa revue Chronique internationale de la Police publiée en français et en anglais dans 66 pays, se fait la porteparole des différentes réalisations effectuées dans cet esprit.

À la fin de l'été 1955, les Nations Unies organisent à leur tour à Genève un premier congrès «en matière de prévention du crime et de traitement des délinquants » qui réunit 500 participants représentants 46 pays. Les travaux sont répartis en trois sections, la dernière étant entièrement dédiée à la prévention de la délinquance juvénile et parmi les intervenants et signataires des documents de travail, on retrouve Paul Villetorte, ainsi que Jacques Siméon, directeur de l'Éducation surveillée au sein du ministère de la Justice. Dans ses conclusions, la section III préconise d'employer essentiellement des mesures préventives qui doivent s'étendre non seulement aux «jeunes qui ont commis un acte considéré comme une infraction à la loi pénale en vertu de la législation de leur pays, mais aussi sur ceux qui en raison de leur condition sociale, risquent de commettre un tel acte ou qui ont besoin d'assistance et de protection ». Elle insiste ainsi sur la notion de " prédélinquance ${ }^{25}$ » et prévoit d'encourager l'institution de services spécialisés de police des mineurs qui seront composés de «fonctionnaires spécialement choisis et préparés pour s'occuper des enfants $»^{26}$.

23 L'impact de ces discussions menées à l'international sur la police française peut se mesurer non seulement par la présence de fonctionnaires gradés, comme Paul Villetorte, à ces réunions mais aussi par l'abondance des articles publiés dans les différentes revues spécialisés de la police, comme la Revue moderne de la Police, Gendarmerie nationale, la Revue de la Sûreté nationale ou encore la revue Liaisons de la Préfecture de police. Ces dernières ouvrent leurs colonnes aux rapporteurs et comptes-rendus de ces rassemblements internationaux et se font l'écho des diverses expérimentations menées en matière de police des mineurs dans les pays plus ou moins voisins comme l'Allemagne, l'Angleterre, le Danemark, les Pays-Bas, la Suède ou même le Japon... Ces chroniques de terrain sont autant d'invites à suivre leur exemple dans le domaine de la prévention.

Par ailleurs, les différentes directions nationales de la police, dans une coordination de plus en plus étroite avec la nouvelle direction de l'Éducation surveillée, répercutent les consignes données à l'international en préconisant par le biais de circulaires la spécialisation d'une partie de leurs personnels et en organisant des sessions de formation pour ces derniers. Le 2 juillet 1953, le ministre de l'Intérieur donne ainsi des directives précises en ce sens aux chefs des services de police placés sous son autorité; ces instructions étant complétées, le 18 mars 1955, par une note de service du directeur général de la Sûreté nationale ${ }^{27}$. De fait, pour la première fois, en mai 1955, quatre commissaires de police et un attaché au cabinet du directeur général de la Sûreté nationale sont désignés pour assister et participer activement aux travaux du $9^{\mathrm{e}}$ stage des juges des enfants organisé au centre de formation et d'études de l'Éducation surveillée à Vaucresson. Dès les premiers jours, des commissions composées de magistrats et de commissaires de police sont constituées pour permettre de débattre de l'assistance réciproque entre policiers et magistrats ${ }^{28}$. Cette première participation donne lieu par la suite, de façon systématique, à l'organisation de "véritables stages » destinés à divers fonctionnaires de police: une session d'études des commissaires de police de la Sûreté nationale du 18 au 23 janvier 1960 ; une pour les commissaires de police de la direction 
des services de police judiciaire du 13 au 17 février 1961; une autre pour les fonctionnaires de la direction de la police judiciaire du 13 au 16 mars 1961 et enfin une autre pour les commissaires de police des services des polices urbaines du 22 au 27 avril 1963. A chacune de ces sessions les exposés théoriques s'accompagnent de discussions de groupes et de visites sur le terrain d'établissements d'observation, de rééducation et de postcure dépendant de l'Éducation surveillée ou d'organismes privés. Elles permettent la rencontre entre intervenants qui d'habitude se côtoient peu, comme le raconte le commissaire principal à Antibes, Victor Ferrari, ayant assisté à la toute première session de janvier 1960 :

«Comme il ne saurait être question de traiter ici des vastes problèmes de l'enfance en état de délit ou de danger mais seulement d'une semaine de séjour au centre de formation de l'éducation surveillée, nous nous satisferons de constater que tout se tient dans les conceptions mises en œuvre, puisque nous avons vu ensemble, dans le même réfectoire, de hauts magistrats, des personnels divers, des élèves éducateurs et des... détenus venus d'une maison d'arrêt voisine, employés comme jardiniers, apparemment aussi libres que nous-mêmes et traités sans discrimination. ${ }^{29}$ "

De même, suite à une expérience menée en novembre 1955 à l'initiative du procureur général de Nancy, M. Reboul, la direction de la gendarmerie et de la Justice militaire adresse à son tour une longue circulaire, le 28 mars 1957, à l'ensemble de ses unités, accompagnée d'un mémento rédigé par M. Durand, juge suppléant du ressort de la Cour d'appel de Nancy. Après un rappel des différentes collaborations possibles entre gendarmeries et tribunaux pour enfants, des mesures spécifiques sont préconisées :

«En principe, dans chaque brigade, un gradé ou gendarme est désigné par le commandant de compagnie pour effectuer les enquêtes sur le mineur : le choix doit s'exercer parmi les sous-officiers possédant une solide expérience de chef de famille ou ayant appartenu à des mouvements de jeunesse (scoutisme en particulier). $»^{30}$

Cette sensibilisation «naturelle» à la question des mineurs est toutefois estimée insuffisante, le souhait étant exprimé que des cycles de conférences, portant notamment sur l'étude des textes légaux et réglementaires relatifs à la protection des mineurs et permettant des échanges de vues avec les assistantes sociales, les délégués à la liberté surveillée et les éducateurs en milieu ouvert, soient organisés, ainsi que des sessions d'études, comme celles réalisées pour les commissaires au centre de Vaucresson. De fait, quatre stages ont effectivement lieu pour les militaires de la gendarmerie les 17-23 novembre 1960, 29 novembre-6 décembre 1961, 6-12 décembre 1962 et 6-14 décembre 1963. Il en va de même pour les compagnies républicaines de sécurité (CRS), quatre sessions d'études ayant été mises en place spécialement pour leurs brigadiers entre 1961 et $1963^{31}$. Un premier survol des programmes de formation proposés par le centre de formation de Vaucresson montre que ce type de sessions se poursuit au moins jusqu'au début des années 1970. Mais seule une étude plus détaillée du nombre de participants à ces sessions permettrait d'en mesurer l'impact réel sur les différents corps de police. A travers les exemples évoqués, il est probable que seul un nombre relativement restreint d'individus ait été concerné à l'image de la faiblesse des effectifs d'agents réellement spécialisés en matière de police des mineurs. Resterait à analyser, en fonction de la position hiérarchique qu'ils occupent à leur retour en poste, comment chacun des participants à ces sessions ont à leur tour diffusé sur le terrain les valeurs inculquées lors de l'apprentissage. 


\section{Une opération « anti-été chaud » avant la lettre}

27 La structuration et l'essor des services de police spécialisés dans les affaires de mineurs semblent s'être cristallisés autour d'une vaste opération conjointe durant l'été 1959, le fameux été des «blousons noirs ». Le projet remonte au 30 avril 1959, lors d'une réunion au ministère de la Justice organisée par le Garde des Sceaux, durant laquelle il est décidé de créer une commission mixte «Justice-Police » réunissant la direction de la Sûreté nationale, la direction de la gendarmerie nationale et la Préfecture de police, en vue de promouvoir et de développer la formation d'une police des mineurs agissant en étroite collaboration avec la chancellerie. Parallèlement, se met en place un groupe spécial, dit "groupe D", chargé de centraliser toutes les informations des services de police et de gendarmerie de France pour constituer une documentation nationale indispensable à l'élaboration des projets intéressant la répression de la délinquance juvénile et la protection de l'enfance en danger physique et moral. La première concrétisation de cette concertation est une expérience pilote, dite "opération vacances », menée du 15 juin au 15 septembre sur la côte des Alpes maritimes dont le dispositif est surtout marqué par l'« unification fonctionnelle » entre les différents services de police :

«C'est à Juan-les-Pins, dans les locaux de l'ancienne mairie annexe d'Antibes que vient donc de s'installer, sous les ordres du commissaire Désiré Yzerman [qui est en même temps chef du "groupe D"], la brigade spéciale de police qui fut constituée de 19 officiers de police et officiers de police adjoints, détachés pour une période de trois mois, soit de la direction de la P.J., soit des services régionaux. Quatre voitures, dont une munie de la radio et reliée au bureau central, deux scooters, du carburant, du matériel de bureau, et un crédit exceptionnel furent mis à leur disposition. La direction des services de la sécurité publique mit à la disposition de la brigade, quatre fonctionnaires motocyclistes de la compagnie C.R.S. de St-Laurent-du-Var pour permettre le contrôle des routes [...] Le personnel de la brigade était scindé en deux groupes, l'un ayant sa base à Juan-les-Pins, l'autre à Nice, chaque groupe étant divisé en deux équipes de quatre fonctionnaires, travaillant alternativement de jour et de nuit. Le service de nuit, sauf modifications imposées par des nécessités particulières, était assuré de 21 heures à 5 heures du matin. Un fonctionnaire de permanence de nuit au commissariat d'Antibes pouvait être touché soit par téléphone, soit par radio. Il disposait du fichier complet de ce service qu'il pouvait consulter très rapidement pour renseigner les équipes de la Brigade opérant dans le département. ${ }^{32}$ »

Le bilan de cette opération est considéré comme suffisamment positif pour la reporter l'année suivante du 15 juillet au $1^{\text {er }}$ septembre 1960 en l'étendant cette fois-ci à l'ensemble du littoral français, soit $5000 \mathrm{~km}$ de côtes, de Dunkerque à Bayonne et de PortVendres à Menton intéressant 25 départements; 186 brigades et postes de gendarmerie, 52 commissariats de police et 6 services régionaux de police judiciaire étant alors mobilisés. Si les «bandes de jeunes, composées soit d'éléments locaux, soit de vacanciers ", font l'objet d'une attention accrue de la part des brigades, qui se prêtent d'ailleurs à des interprétations pseudo-scientifiques et psychologisantes sur leurs comportements, en revanche dans les rapports effectués par ces premières brigades côtières, on ne trouve aucun écho du cri d'alarme lancé à la même période par les médias autour du phénomène « blousons noirs $»^{33}$. Bien au contraire, les incidents qui dégénèrent parfois en pugilats sont banalisés et très souvent remis à leur juste mesure :

«Les jeunes gens du pays rôdent aux alentours [des campings] pour rencontrer les jeunes filles du camp. Il en résulte souvent des rixes entre eux et les garçons du 
village de toile qui "prétendent défendre leur terrain de chasse". Des conflits naissent également avec les jeunes gens locaux du seul fait de la différence d'origine géographique. Il s'en suit des provocations entre groupes qui dégénèrent en "bagarres". L'incident de Cannes est de cette nature. Ce fut un banal exemple de la "guerre des boutons". Un jeune homme de Courbevoie âgé de 18 ans, est agressé et blessé par des jeunes locaux en sortant du cinéma. Il est en vacances avec un groupe de jeunes de sa région, en camping. Il court aussitôt faire appel à eux et commande une "expédition punitive" contre la bande dont il vient d'être victime. Vêtus de blousons de cuir, coiffés de casques de motocyclistes, armés de gourdins et de ceinturons, ils traversent la ville sur le "sentier de guerre". Le groupe menaçant, interpellé par des gardiens de la Paix se rebelle; un sous-brigadier est frappé et tombe en se luxant le poignet. (Le lendemain, un grand quotidien parisien rapporte en titre qu'un agent a été "poignardé »). [...] Un autre incident fit beaucoup de bruit, trop de bruit dans la presse : à Bandol, ville située hors des limites de la mission, dans un café-dancing, une rivalité au sujet de jeunes filles en vacances mit aux prises des jeunes du cru à 21 toulonnais venus dans quatre voitures en "expédition punitive". " $^{34}$

Le bilan des "opérations vacances » dressé par Jean Susini, commissaire principal à la Sûreté nationale est en décalage avec le battage médiatique autour des affaires où sont mêlées les bandes. Il déclare ainsi que «en cherchant d'authentiques manifestations de criminalité ", l'enquête effectuée en 1959 "rencontra surtout des manifestations de désorganisation sociale.» Il rappelle par ailleurs que si les policiers intervenaient au moment même de leur constitution, la plupart des bandes avaient été disloquées ${ }^{35}$. Les principaux incriminés sont souvent les gérants de camping qui « jouent le rôle de maquis dans lesquels peuvent se dissimuler des individus peu recommandables ». Il est alors préconisé une réglementation plus stricte des camps et séjours de jeunes tout en signalant que la lutte « contre l'oisiveté et l'abandon moral des jeunes » devrait être aussi épaulée par une organisation meilleure, voire plus disciplinée, de leurs loisirs.

Malgré le changement de regard porté sur les sociabilités juvéniles qui apparaît même parfois en décalage avec le discours alarmiste de l'époque sur les bandes et se traduit par une volonté de déployer une action préventive, prenant le pas sur la répression, la notion même de prévention telle qu'elle est développée dans les comptes rendus d'activité des premières brigades est encore étroitement liée aux fonctions de maintien de l'ordre exercées traditionnellement par la police. Il s'agit en effet surtout d'action de fichage, de repérage, de contrôle et de patrouille, voire même d'interpellations, même si ces dernières se concluent la plupart du temps par une simple admonestation. Les agents mobilisés misent moins sur la discrétion des ressources humaines déployées - «leur présence sur la côte » ayant été «vite repérée » et un «tam-tam secret » ayant «mis en alerte le plus grand nombre » - que sur l'impact dissuasif et la force d'intimidation de l'uniforme. L'objectif principal est d'arriver à contenir les effets négatifs de cette migration saisonnière massive des adolescents en établissant la « liste des points les plus sensibles du littoral grâce à l'examen des statistiques rassemblées ». Une surveillance accrue de tous les lieux de rassemblement ou de séjour des jeunes est ainsi organisée par des rondes régulières aux abords des cafés, kermesses de jeux, disquaires, hôtels, pensions, camps de jeunesse, terrains de campings, centres de vacances; en particulier les "pôles d'attractions nocturnes" (dancings, music-halls, cabarets, boîtes homosexuelles)... ainsi que la nuit sur la route de la côte et les plages. 


\section{De la police des loisirs aux policiers « moniteurs »}

31 Les deux « opérations vacances » de 1959 et 1960 marquent donc un tournant dans la conception du rôle des brigades de mineurs en particulier dans le domaine du loisir. Jusqu'alors l'intervention des policiers se limitaient à la traque systématique de tous les lieux où les jeunes pouvaient s'abandonner à l'oisiveté ou aux « mauvais loisirs ", dont la publicité pernicieuse et les enseignes clinquantes les attiraient tels des papillons aveuglés par la lumière des néons. Leur mission de vigilance consistait donc à un contrôle strict, une surveillance continue des lieux publics et un rappel à l'ordre pour la bonne application des textes de lois, de plus en plus nombreux, à venir réglementer l'accès et la fréquentation de ces espaces de distraction et autres lieux de tentation comme les grands magasins. La conférence effectuée en 1955 durant le camp-école national des scouts de France à Jambville pour les éducateurs de l'enfance inadaptée, par Fernand Zamaron, commissaire divisionnaire et chef de service de la protection des mineurs à la Préfecture de police, est représentative de cette vision des choses :

« Le mot loisir en lui-même serait assez rassurant. Il évoque le calme, la détente, la satisfaction légitime des goûts, la compensation du travail et de l'effort. C'est cependant au cours de ses loisirs que le jeune pourra mal faire ou se nuire et qu'il devra donc être surveillé et protégé. Dans cet exposé il ne sera question que des risques puis des moyens dont disposent les services de police d'une vaste agglomération pour les écarter et les atténuer. ${ }^{36}$ »

Il liste alors de façon systématique les limites ou les interdits posés par une législation de plus en plus tatillonne à l'égard des fêtes foraines, le cinéma, les kiosques à journaux, les débits de boissons, les bals, les "caves", les jeux d'argent... Bien qu'il évoque en conclusion la nécessité de créer un contrepoids à ces endroits irrésistibles, en aménageant des "sortes de stations-services du loisir ", certaines d'entre elles pouvant être organisées ou patronnées comme aux États-Unis par des services de police, il ne s'agit encore que d'une déclaration d'intention. L'exemple étranger des clubs de jeunes aménagés ou animés par des policiers, pourtant largement commenté et valorisé dans les différentes revues de la police se heurte en effet dans un premier temps à une méfiance des services de sécurité français, comme le montre encore l'intervention faite par le capitaine de gendarmerie Collet lors d'une conférence donnée le 27 juin 1957 à l'Institut national d'éducation populaire de Marly-le-Roi :

\footnotetext{
« Peut-être seriez vous tentés de penser qu'en matière de protection de l'enfance, la gendarmerie est en retard d'une guerre et qu'elle eut été bien inspirée d'imiter l'exemple de la Suède, du Canada et du Danemark où la police a créé des clubs de jeunes. Il m'apparaît hasardeux de vouloir ainsi importer des institutions étrangères qui se rattachent à un contexte civique, social et psychologique différent du nôtre. Je ne pense pas personnellement, qu'en France, les relations police-public soient assez mûres pour engendrer sans douleur des initiatives aussi audacieuses, et M. le conseiller Chazal avait raison de dire récemment qu'il est encore prudent de ne pas demander à la police de diriger des "boys clubs". $»^{37}$
}

Les opérations menées durant les étés 1959 et 1960, précédées de peu par la première expérience de surveillance des baignades par des CRS tentée en 1958 sur 20 plages des côtes bretonnes en collaboration avec les hospitaliers sauveteurs bretons, donnant naissance aux maîtres nageurs sauveteurs (MNS), font sauter les dernières résistances. Tandis que, fort de leur succès remporté sur les côtes bretonnes, le nombre de CRS-MNS est porté à 150 en 1959, on assiste progressivement entre 1960 et 1964 à la multiplication 
de "clubs vacances" et autres expériences de "centres de loisirs organisés » où les policiers décident d'abandonner leur rôle de gardien de la Paix en uniforme pour investir pleinement celui d'animateur. En 1965, Jean-Marie Dupont publie ainsi deux articles dans Le Monde sous le titre évocateur : "Des policiers moniteurs de jeunesse », dans lesquels il évoque l'extension de ce type d'initiatives :

«Certains policiers ont estimé qu'il fallait s'attaquer à la racine du mal: le désœuvrement de ces adolescents en liberté et sans argent. Dès 1962, un centre nautique pour les jeunes était lancé à Dinard par le commissaire de police, avec le concours de la direction de la population et de la municipalité. L'été suivant le centre se transformait en école de voile, et un club de loisirs animé par des moniteurs de la sûreté nationale (gardiens des CRS et des polices urbaines) rassemblait environ cent cinquante adolescents. Un autre était créé la même année à Royan. En 1964, plusieurs commissaires de police du Tréport à Hyères prenaient des initiatives similaires tandis que les CRS tentaient à leur tour une expérience de centre de vacances pour les jeunes à Hossegor. Le succès de ces différentes tentatives s'est traduit cet été par la mise en place de vingt-huit centres de loisirs, quatorze contrôlés par les CRS, quatorze par les polices urbaines qui ont touché au total plus de cinq mille adolescents. Et il semble que ce ne soit qu'un début. Que penser de cette transformation de policiers en promoteurs et animateurs de clubs de jeunes ? S'agit-il d'encadrer la jeunesse, de prévenir la délinquance juvénile ou d'assumer un rôle de suppléance dans l'organisation des loisirs? N'y a-t-il pas risque de confusion dangereuse à confier à des fonctionnaires chargés d'assurer l'ordre public des tâches de moniteurs de jeunesse ? "

Malgré les réserves exprimées, il témoigne avec une certaine fascination de sa visite à l'un d'entre eux :

«À l'extrémité ouest de la plage du Lavandou une banderole annonce : "Centre de loisirs des jeunes" avec à côté la mention "Sûreté nationale-CRS", de l'autre le nom de la station. Une quinzaine de jeunes jouent au volley. Les deux tables de pingpong et de baby-foot placées sous un auvent sont occupées. Quelques garçons et filles allongés près d'une baraque, qui sert de vestiaire et d'entrepôt pour le matériel, discutent en écoutant des disques. Une dizaine d'autres sont partis en mer avec le moniteur de voile. Au total, ils sont environ quatre-vingt présents en permanence de 9 heures le matin à 8 heures le soir, mais il y a plus de cent cinquante inscrits. [...] "Ici", ce n'est plus l'entassement, la solitude au milieu de la foule, mais une sorte de "domaine", où les quinze à vingt ans sont entre eux. Outre cette possibilité de se retrouver entre copains, ce que les jeunes déclarent apprécier beaucoup c'est l'absence de contrainte. Le centre n'est pas un camp militaire. Chacun arrive et part quand il le désire et si, entre deux parties de volley-ball, quelqu'un a envie de se reposer en écoutant un disque, personne ne l'en empêche..."

Si le journaliste du Monde semble prêt à céder à ce qu'il qualifie lui-même "d'opération charme ", il ne manque pas de s'interroger sur l'efficacité de ce type d'expérience pour le contrôle de la délinquance juvénile :

«Dans les deux centres visités nous n'avons pratiquement pas rencontré de jeunes délinquants ou "perturbés ", mais des adolescents en général de «bonne famille » qui tout simplement s'ennuyaient. Bien sûr, l'oisiveté et les tentations de tous ordres que rencontrent les jeunes dans une station estivale peuvent les mener à commettre des délits quelle que soit leur origine. Les exemples d'«exploits» de "blousons dorés" sont là pour le confirmer. Mais on peut se demander si l'action $\mathrm{du}$ policier ne doit pas viser d'abord à sauver ceux qui sont au bord de la délinquance. ${ }^{38}$ 
articles de Jean-Marie Dupont ont suffisamment d'impact pour faire réagir certains organismes de jeunesse comme la Jeunesse au plein air (JPA) ${ }^{39}$, qui s'inquiète de ce nouveau "rôle de suppléance " assumé par les policiers des brigades des mineurs et rappelle qu'« il faut prévoir un encadrement de qualité » que seuls peuvent garantir les mouvements de jeunes en participant à la formation officielle des moniteurs de la jeunesse diplômés d'État ${ }^{40}$. Il est de plus ajouté que la position reconnue des mouvements de jeunesse dans le domaine des loisirs, pourrait être étendue aux jeunes des «bandes ", ceux que l'on surnomme les « inorganisés » à condition qu'ils bénéficient d'une aide de l'État plus conséquente et qu'ils puissent faire appel non pas aux militaires de la gendarmerie mais à ceux du contingent, parmi lesquels nombreux sont ceux qui ont « les titres et les qualités pour apporter pendant leurs congés d'été leurs concours à des centres de vacances pour adolescents. ${ }^{41}$ »

Peu touchés en apparence par ces critiques, les revues de la police se font l'écho des très nombreuses initiatives similaires menées non seulement avec l'appui des ministères de l'Intérieur et de la Justice, mais aussi avec celui de la Jeunesse et des Sports. Henri Paoli, commissaire principal de la Sûreté nationale à Paris évoque ainsi la multiplication des réalisations entre 1963 et 1965, donnant naissance à des associations dont les appellations cherchent à favoriser l'adhésion de tous : «Pieds dans l'eau » pour Antibes, «VacancesArdennes » pour Mézières-Charleville-Mohan, «Club de jeunes » à Boulogne, «Club des loisirs » à Saint-Raphaël, Hyères, "vacances sourires » à Eu, le Tréport, Mers, dont les histoires particulières restent encore à faire. Il évoque même un prolongement de cette action qui ne se limiterait pas à la période des vacances :

"L'avenir doit conduire le policier urbain à une intégration plus parfaite encore à l'appareil socioéducatif mis en place pour les jeunes et appelé à évoluer profondément. Une réalisation à la dimension d'un quartier a été développée à Marseille. Une autre faite à l'initiative du ministère de la Jeunesse et des Sports d'un genre un peu différent se déroule actuellement dans une ville de l'intérieur. Autour d'un club de jeunes créé dans un ensemble groupant 10.000 habitants, et dont le sort était compromis par un manque de moyens, se sont trouvés rassemblés : le maire de la ville, le directeur des caisses d'allocations familiales, le directeur départemental de la Jeunesse et des Sports, un délégué aux maisons de jeunes et de la culture, le directeur d'un foyer de jeunes travailleur, les dirigeants des mouvements de jeunesse et des policiers urbains. Tous étaient désireux d'effectuer un travail en profondeur ayant une valeur sociale véritable. Le principe de la création d'une maison de jeunes et de la culture a été retenu comme moyen idéal de répondre à leurs préoccupations. Le projet est en voie de création. Dirigeants de mouvements de jeunes, membres de la Jeunesse et des Sports, directeur du foyer de jeunes travailleurs, membres bénévoles, policiers urbains en seront les animateurs. $42 »$

Si l'image du CRS-maître nageur a perduré, qu'en est-il de ces expériences de clubs de loisirs qu'il reste à recenser de façon plus exhaustive ? Pourquoi reste-t-il si peu de traces de leur fonctionnement et à partir de quelle date ont-ils commencé à péricliter ? Ont-ils été victimes comme l'ensemble des colonies de vacances de la pénurie des effectifs et des subventions à la fin des années 1960 ou bien la concurrence exercée par la lente montée de la fonction des animateurs socioculturels a-t-elle fini par en sonner le glas ? Encore une fois des travaux de recherche s'imposent, fonds d'archives à l'appui. 


\section{$\mathrm{Ni}$ « croquemitaines » $\mathrm{ni}$ « matraqueurs », mais gardiens de l'ordre tout de même}

39 Avec le recul, cette volonté partagée par l'ensemble des corps de police de mettre l'accent sur les activités de prévention, à une époque où la société française semble avoir si peur de sa jeunesse et de ses sociabilités - stigmatisées en les limitant au phénomène des «bandes "-, ne peut que nous interroger. D'autant que la notion de prévention telle qu'elle est déclinée durant les années 1950-1960, par les services de police cherche à se démarquer de plus en plus nettement du seul maintien de l'ordre.

Elle passe tout d'abord par une remise en cause en profondeur des méthodes traditionnelles quand il s'agit d'interventions auprès de mineurs, dans une volonté explicite d'accommoder les procédures aux exigences de la personnalité du jeune délinquant ; l'idée étant de devenir un des rouages de la nouvelle justice des mineurs telle qu'elle a été pensée dans l'ordonnance de 1945. L'interpellation en public, l'usage des menottes, la conduite au poste, le dépôt dans la chambre de sûreté, les interrogatoires musclés dans la seule quête de la vérité sont unanimement déconseillés, tandis que le tact, la prudence, la discrétion, la psychologie, la politique de la main tendue, le « sens du social » sont mis à l'honneur. Si ces consignes sont répétées comme un leitmotiv durant toute la période considérée on ne peut que se questionner sur la façon dont elles ont été entendues parmi les acteurs de terrain. Si les brigades ou services spécialisés semblent effectivement s'être multipliés, comment étaient-ils perçus par les collègues qui n'en faisaient pas partie ? Ont-ils été « contaminés » par cet «élan pour la prévention » tant célébré dans les revues professionnelles?

41 Ne peut-on penser qu'il ne s'agit là que d'initiatives ponctuelles, très minoritaires dont l'enjeu est avant tout publicitaire, une sorte d'affichage? Les brigades des mineurs ne seraient-elles dans ce cas que des vitrines - une sorte d'arbre qui cache la forêt des abus et des négligences du reste de l'institution ? Les articles parus dans les revues spécialisées ou les circulaires ne témoigneraient-ils que du développement d'une idée, d'une intention, d'une préconisation? La question mérite d'être posée et confrontée aux pratiques réelles sur le terrain (qui doivent être documentées par une étude du travail concret de ces brigades tel qu'il apparaît dans les archives et les récits de vie), même si certains documents et rapport publiés dans ces revues de par leur aspect austère, sans enjolivures, laisse à penser qu'il ne s'agissait pas que d'effets de manche.

La prévention dans la police s'est aussi conjuguée, nous n'avons pas pu ici le développer, avec prévention routière, avec l'installation, dès le milieu des années 1950, de pistes d'épreuve de circulation pourvues de voitures à pédales et régentées par un gendarme ou un policier avec la participation active des élèves ; pistes présentées dans les divers salons de l'enfance et diffusées dans les écoles.

La prévention telle qu'elle est déclinée par les services de police finit même par rejoindre au début des années 1960 celle pratiquée par certaines associations de prévention spécialisée et éducateurs de rues, centrée sur un quadrillage du territoire et la mise en place de clubs offrant des loisirs sains, attractifs et bien encadrés et mettant l'accent sur les exploits sportifs. Une différence de fond subsiste cependant : celle du mandat, de la distance à respecter par rapport à la mission assignée et du pouvoir d'intervention. 
Comme le rappelle Paul Villetorte, «Il ne s'agit pas de travestir le policier en travailleur social $»^{43}$ au risque de le mettre en difficulté :

«Le danger est grand de s'incorporer directement, même sous le couvert de l'anonymat dans des groupes de jeunes prédélinquants et délinquants car le policier ne pourrait guère faire la sourde oreille aux propos échangés devant lui, et se rapportant à des actes de délinquance déjà commis ou sur le point de l'être. Il devra nécessairement intervenir sous peine de faillir à son devoir professionnel, de manquer à l'exercice de ses fonctions..$^{44}$ "

Cette situation de porte-à-faux explique sans doute les valses-hésitations dans la présentation de l'idéal-type du " policier mineur » évoqué dans les revues de la police; si l'on cherche à se démarquer de l'image du «croquemitaine » ou du « « croquemitaine à bicorne » ou encore du «matraqueur", il ne saurait être question de renier la fierté de l'uniforme. S'il s'agit de « donner le pas à la prévention sur le tableau de chasse tangible et spectaculaire $~^{45}$, on ne peut faire fi de l'attente d'une certaine efficacité en matière de contrôle et de fichage policier. Malgré cette ambivalence ou grand écart, on ne peut qu'être frappé par l'enthousiasme manifesté et la propagation de ces idées de prévention durant les années 1950-1960 au sein des différents corps de la police alors que le contexte de l'époque et la campagne médiatique semblaient demander plus de sévérité. Ces initiatives qui peuvent être pistées jusqu'au début des années $1970^{46}$, encore faudrait-il en suivre l'évolution de plus près, semblent par la suite tomber totalement dans l'oubli.

Il est même possible de parler d'amnésie alors que la question des relations entre les jeunes et la police se pose avec la même acuité, trouvant à nouveau un relais alarmiste dans les médias. À ce silence des services spécialisés de la police répond le silence des chercheurs qui jusqu'à maintenant se sont désintéressés de cette histoire, qui est restée cantonnée dans les revues spécialisées sous la plume des professionnels ${ }^{47}$. Que sont devenus les brigades et services spécialisés dans les affaires de mineurs? Pourquoi sontils si silencieux dans une actualité brûlante où l'affrontement entre jeunes des cités et force de l'ordre semblent atteindre son paroxysme? Qu'en est-il des exigences de formation, de sélection, d'échanges de vues avec les autres intervenants, posées à l'époque comme condition de la réussite de ce type d'opération? Pourquoi la prévention n'est-elle plus à l'ordre du jour dans les services de la police où semble dominer une escalade dans la répression?

Les brigades des mineurs actuelles ne s'intéressent plus, semble-t-il, qu'aux mineurs victimes et non aux mineurs auteurs; elles se consacrent désormais plus particulièrement aux violences physiques et sexuelles faites aux mineurs. Encore faut-il en dater précisément le tournant qui semble s'opérer dans les années 1970. Geneviève Pruvost suggère l'hypothèse qu'entre le moment où les brigades des mineurs sont créées et le moment de la découverte de l'inceste et des violences sexuelles (cause de certaines fugues de mineurs poursuivies par les brigades des mineurs) dans les années 1980, un espace s'est dégagé pour penser à la prévention, mais que cet espace s'est aussitôt réduit quand les crimes sexuels ont émergé sur la scène publique et qu'il a fallu affecter la majorité des effectifs au traitement de ces affaires autrefois minorées. Elle propose aussi l'idée que la prévention s'est déplacée aux commissariats de sécurité publique et non plus aux brigades spécialisées (contenant des officiers) qui ne peuvent balayer le terrain comme le font la masse des gardiens de la paix : les mineurs auteurs sont en effet appréhendés par les policiers de la police secours et c'est eux qui vont prendre en charge par des opérations de sensibilisation dans les collèges et lycées par exemple le travail préventif. Il 
s'agirait ainsi moins d'une disparition du concept de prévention que d'un déplacement au sein de l'organisation des forces de police qui lui sont affectées : déplacement vertical (des inspecteurs aux gardiens de la paix), déplacement horizontal (de la police judiciaire à la sécurité publique). Elle évoque enfin la nécessaire distinction à établir entre la Préfecture de police parisienne (seule police municipale qui a subsisté après guerre, disposant jusqu'en 1966 de ses propres concours, de sa propre formation et de sa propre hiérarchie) et la Sûreté nationale. En matière de brigade des mineurs, les pionniers sont en effet parisiens et ils disposent d'une brigade tout à fait originale, empreinte, selon les témoignages, d'une atmosphère religieuse qui conditionne largement le rapport au travail et à la prévention. La Sûreté, aussi bien en termes d'effectifs (proportionnellement à Paris) que de réflexion, est en situation de rattrapage ou d'imitation. Il faudrait alors travailler sur les transferts de compétence et de pratique. D'un côté, à Paris, le gros de l'activité de la brigade des mineurs reste centré sur l'enquête sociale, le personnel ayant souvent un diplôme d'assistante sociale ou d'infirmière, même si l'obligation d'être dotée de ce diplôme n'en est plus une à partir des années 1950. Et, du côté de la Sûreté nationale, les services sont composés d'inspecteurs ordinateurs, qui ne sont pas formés comme ont pu l'être les inspecteurs de la brigade des mineurs parisienne ; on attend d'eux qu'ils soient volontaires et paternalistes. La professionnalisation est d'un côté prise au sérieux, de l'autre à peine formalisée ${ }^{48}$.

\section{NOTES}

1. La fureur de vivre (Rebel Without a Cause), film américain réalisé par Nicholas Ray et sorti en 1955.

2. Hélène Campinchi-Landry (1898-1962), avocate à la cour d'appel de Paris, co-fondatrice du service social pour l'enfance en danger moral de Paris, administratrice de l'Union des sociétés de patronage, présidente de la commission qui aboutit à la rédaction de l'ordonnance du 2 février 1945 ; épouse de César Campinchi (1882-1941), ancien Garde et Sceaux et promoteur d'un projet de loi en 1937 concernant le droit et la protection de mineurs.

3. Henri Joubrel (1914-1983) fait une brève carrière d'avocat, puis de magistrat, avant de se spécialiser à partir des années 1940 en tant que permanent du mouvement scout des éclaireurs de France dans la défense d'une meilleure prise en charge de la jeunesse « dite coupable ", promouvant notamment la professionnalisation et le perfectionnement des éducateurs spécialisés.

4. Cette communication est publiée à deux reprises dans la Revue moderne de la police, $\mathrm{n}^{\circ}$ 19, juillet-août 1956 et dans la revue Liaisons de l'Association nationale des éducateurs de jeunes inadaptés, $n^{\circ} 20$, octobre 1956, p. 10-11.

5. Alors que la majorité pénale est passée de 16 à 18 ans en 1906, la majorité civile reste fixée à 21 ans jusqu'en 1974 date à laquelle elle rejoint la limite de la majorité pénale, en étant abaissée à 18 ans. 
6. Geneviève Pruvost, De la "sergeote» à la femme flic, une autre histoire de l'institution policière (1935-2005), Paris, La Découverte, 2008, 309 p. et le chapitre 1 « Au nom des vertus féminines (1935-1968) » de sa thèse de doctorat: L'accès des femmes à la violence légale. La féminisation de la police (1935-2005), sous la direction de Rose-Marie Lagrave, EHESS, 2005.

7. Audrey Ambraise, Les femmes dans la Police à Lyon de 1937 à 1956, mémoire de maitrise d'histoire sous la direction de Sylvie Schweitzer, Université Lyon 2, 2000, 143 p.; Des policières à Lyon. Etude sur les métiers des femmes dans la Police de Lyon et sa région 1937-1968, mémoire de DEA d'histoire sous la direction de Sylvie Schweitzer, Université Lyon 2, 2002, $128 \mathrm{p}$.

8. Voir les travaux de Marie Vogel, notamment, «Police et espace urbain: Grenoble 1880-1930 ", Revue d'Histoire Moderne et Contemporaine, Espaces policiers, XVII ${ }^{\mathrm{e}}-\mathrm{XX}{ }^{\mathrm{e}}$ siècles, vol. 50, n 1, janvier-mars 2003, p. 126-144.

9. Léonie Chaptal, Service social et assistantes sociales, Paris, SPES, 1933; Roger-Henri Guerrand, Marie-Antoinette Rupp, Brève histoire du service social en France (1896-1976), Toulouse, Privat, 1978; Brigitte Bouquet, Christine Garcette, Assistante sociale aujourd'hui, Paris Maloine, 2009.

10. Fernand Zamaron (commissaire divisionnaire, chef des services de la protection des mineurs et de la natalité à la Préfecture de police), «L'organisation de la protection des mineurs ", Rééducation, 1949, n 13, p. 5-28; Camille Bouvier (commissaire de Police à la Préfecture de police de Paris, adjoint du commissaire divisionnaire Lefevre, chef brigade de protection des mineurs de la police judiciaire de la préfecture de police, «La brigade de protection des mineurs", Revue internationale de police criminelle, $\mathrm{n}^{\circ} 177$, avril 1964, p. 103-108.

11. Fernand Zamaron, Police des mineurs, grande police, Paris, édition à compte d'auteur, $1958,178 \mathrm{p}$.

12. M. Lefeuvre (commissaire divisionnaire à Paris, chef de la brigade de protection des mineurs de la direction de la police judiciaire), «Contribution de la police parisienne au traitement de l'état de prédélinquance juvénile ", Liaisons, bulletin d'information de la préfecture de police, $\mathrm{n}^{\circ} 101,20$ octobre 1965, p. 1-8.

13. Guy Le Lan (inspecteur de Police à la brigade des mineurs du Havre, lauréat du grand concours d'idées organisée par la revue internationale de criminologie et de police technique de Genève), "Comment lutter contre la délinquance juvénile? », Revue de la sûreté nationale, $\mathrm{n}^{\circ} 23$ août-septembre 1959, p. 13-16

14. Une comparaison serait à établir avec le cas anglais grâce au travail de Louise Jackson, Women Police. Gender, Welfare and Surveillance in the Twentieth Century, Manchester University Press, 2006, 220 p. Les femmes policiers en Angleterre semblent elles aussi avoir été recrutées parmi les étudiantes en travail social en mettant l'accent sur le caractère social du métier.

15. Qui se différencie des autres personnels, techniques et administratifs.

16. Fernand Zamaron, «L'organisation de la protection des mineurs », Rééducation, 1949, doc. cit.

17. Rapport annuel sur le fonctionnement du tribunal pour enfants et adolescents de la Seine pendant l'année 1931, Melun, Imprimerie administrative, 1932, p. 19-20. 
18. «La police au service de l'enfance ", Cahiers de l'enfance, $n^{\circ} 8$, juin-juillet 1954 , p. 53-55; Lucette Bihoux, « Police et délinquance », Cahiers de l'enfance, $n^{\circ} 12$, décembre 1954, p. 33-36.

19. L'association nationale des éducateurs de jeunes inadaptés est créée en 1947 et publie son bulletin Liaisons à partir de 1951.

20. Louis Casali, «Avec la police de Cherbourg... à l'heure de la prévention », Liaisons, $\mathrm{n}^{\circ}$ 21, janvier 1957, p. 14-21.

21. Paul Coupet, «Brigade des mineurs et police moderne ", Revue moderne de la police, $\mathrm{n}^{\circ}$ 43, juillet-août 1960, p. 22-26.

22. Geneviève Montagne, Une création toulousaine, la brigade des mineurs, thèse de doctorat, Droit, Toulouse, 1956, 78 p.

23. Lors de la $21^{\mathrm{e}}$ session, juin 1952 à Stockholm, rapport et résolution $\mathrm{n}^{\circ} \mathrm{XI}$; de la $22^{\mathrm{e}}$ session de juin 1953 à Oslo, rapports 3 et 5 ; et de la $23^{\mathrm{e}}$ session d'octobre 1954 à Rome, résolution IX.

24. OIPC-Interpol, "Délinquance juvénile et police", Revue internationale de police criminelle, $\mathrm{n}^{\circ} 143$, décembre 1960, p. 317-321.

25. Cette notion va à l'inverse de la philosophie du droit pénal français qui pose comme règle la présomption d'innocence, alors que dans le cas des mineurs semble prévaloir la présomption de culpabilité.

26. «Premier congrès des Nations Unies en matière de prévention du crime et de traitement des délinquants Genève, 22 août - 3 septembre 1955 ", Revue moderne de la Police, vol. 3, n 15, nov-déc 1955.

27. Circulaire et note de service évoquée dans «La spécialisation et la formation des personnels de la police et de la gendarmerie ", Rapport annuel de l'éducation surveillée, 1963, p. 178-179.

28. Maurice Boutemy (commissaire principal, chef de la sûreté à Toulouse), «Un exemple de collaboration entre les commissaires de police et les juges des enfants », Revue moderne de la police, $\mathrm{n}^{\circ} 14$, septembre-octobre 1955, p. 32-34.

29. Victor Ferrari, «A Vaucresson. Session des commissaires de la sûreté nationale au centre de formation et d'études de l'éducation surveillée ", Revue de la Sûreté nationale, $\mathrm{n}^{\circ}$ 30, avril 1960, p. 3- 6 .

30. Circulaire diffusée intégralement dans le Rapport annuel de la direction de l'Éducation surveillée, 1961, p. 135-182.

31. "La spécialisation et la formation des personnels de la police et de la gendarmerie ", Rapport annuel de la direction de l'Éducation surveillée de 1963, p. 173-216.

32. Cette expérience pilote est très abondamment commentée et décrite dans les différentes revues de la police, voir par exemple : «Délinquance juvénile. Une expérience de la Sûreté nationale dans les Alpes-Maritimes ", Revue de la sûreté nationale, $\mathrm{n}^{\circ} 28$, février 1960 , p. 3-12

33. Désiré Yzerman, « Répression de la délinquance juvénile et protection de l'enfance en danger ", Rapport sur l'expérience été 60 faite sur l'ensemble du littoral français du 15 juillet au 31 août 1960, ministère de l'Intérieur, direction générale de la sûreté nationale, direction des services de police judiciaire, Paris, le 13 décembre 1960, 29 p., fonds ENPJJ-Roubaix.

34. « Délinquance juvénile. Une expérience de la Sûreté nationale... », art. cit., p.9. 
35. Jean Susini, «F. chronique de police. Deux essais de prévention de la délinquance juvénile par la police française", Revue de science criminelle et de droit pénal comparé, nouvelle série, $n^{\circ} 4$, octobre-décembre 1960, p. 698.

36. Fernand Zamaron, «Les loisirs des jeunes dans les grandes villes. Rôle et action des services de police à la préfecture de police ", Compte rendu des Journées d'étude et d'information pour les éducateurs de l'enfance inadaptée, Quartier général des Scouts de France, 1955, 14 p., fonds ENPJJ-Roubaix.

37. Capitaine Collet, "Les orientations éducatives de la gendarmerie en matière de protection de l'enfance ", conférence donnée le 27 juin 1957 à l'Institut national d'éducation populaire, Marly-le-Roi, fonds ENPJJ-Roubaix.

38. Jean-Marie Dupont, premier article portant le sous-titre : "Les CRS chez les copains », Le Monde, 2 septembre 1965.

39. La Fédération nationale des œuvres laïques de vacances d'enfants et d'adolescents est fondée en 1938, regroupant notamment la Ligue de l'enseignement, la Fédération générale des pupilles de l'enseignement public, l'Hygiène par l'exemple ; elle prend plus tard le nom de JPA du nom de sa campagne de timbres vendus au profit des œuvres.

40. Diplôme existant depuis 1947, Françoise Tétard, «Moniteur de colonies de vacances. Le diplôme, pour unifier un secteur très tendance ", Loisirs Education, revue de la JPA, $\mathrm{n}^{\circ}$ 391-392, février-mars 2002, p. 14-15.

41. « Des policiers moniteurs de la jeunesse », commentaire des articles du Monde des 2 et 3 septembre 1965, revue de la Jeunesse au plein air, n 127, septembre-octobre 1965.

42. Henri Paoli, «Le policier et l'enfant », Revue de la Sûreté nationale, juin-juillet 1966, n 63, p. 13-15.

43. Paul Villetorte, «L'apport de la Police à l'observation des mineurs en milieu ouvert en France ", Chroniques internationales de police, $\mathrm{n}^{\circ}$ 48, mai-juin 1961, p. 9-16.

44. Lefeuvre (commissaire divisionnaire à Paris, chef de la brigade de protection des mineurs de la direction de la police judiciaire), «Contribution de la police parisienne au traitement de l'état de prédélinquance juvénile », Liaisons, bulletin d'information de la préfecture de police, $\mathrm{n}^{\circ}$ 101, 20 octobre 1965, p. 1-8.

45. Victor Ferrari, "Prises de contact entre la police et les jeunes ", Revue de la Sûreté nationale, $\mathrm{n}^{\circ} 35$, décembre 1960, p. 11-15.

46. L'expérience du Bureau d'accueil de la jeunesse, BADJ mis en place à la préfecture de Paris dès 1967 reste ainsi encore à analyser.

47. A part les quelques pistes lancées par les chercheurs du Centre interdisciplinaire de Vaucresson comme par exemple l'article de Jean-François Gazeau, «Réflexions sur la brigade des mineurs ", Droit de l'enfance et de la famille, Vaucresson, CFRES, 1977/1, p. 85-91 ; ou celui de Daniel Condaminas, "Le service départemental des mineurs du Val de Marne", Cahiers du CRIV, $\mathrm{n}^{\circ}$ 4, janvier 1988, p. 103-106 ou encore celui de Pierre Lascoumes et Hartwig Zander, « Débat : Jeunesse, police et prévention », Déviance et Société , Genève, 1982, vol.6, n 2, p. 185-187.

48. Je remercie chaleureusement Geneviève Pruvost, qui a accepté de lever son anonymat de relectrice pour que je reprenne à mon compte ses remarques pertinentes sur les dernières évolutions de la brigade des mineurs, tout en assumant l'entière responsabilité des libertés d'interprétation que j'ai pu en faire. 


\section{RÉSUMÉS}

Alors que les affrontements violents entre jeunes des quartiers et police font la une de l'actualité, au point de parler de divorce et de la nécessité de renouer un dialogue, il y a un corps étrangement absent et silencieux dans cette scène médiatique : la Brigade des mineurs. Créée en 1958-1959 et faisant suite au service de protection des mineurs du début des années 1950, luimême héritier du corps spécialisé des Assistantes de police apparu au milieu des années 1930, la Brigade avait été pourtant pensée au départ dans un objectif de prévention de la délinquance juvénile et ce n'est que bien plus tard qu'elle prendra en charge les enfants victimes. A ce silence actuel surprenant de ce corps spécialisé ayant plus de 50 années de pratique, correspond le silence des historiens. A part quelques travaux sur les femmes-policières réalisées par certaines historiennes du courant de l'histoire des femmes, il n'existe à ce jour aucune analyse de la création et des expériences menées sur le terrain par ce corps de police spécifique. Les revues de la police, les revues spécialisées du travail social et de nombreux rapports et brochures des années 1950-1970 rendent pourtant compte des actions projetées et réalisées tant en France que dans les pays voisins et qui semblent aujourd'hui avoir été totalement oubliées.

At a time when the clashes between the police and youths from the suburbs are headline news and people talk about divorce and the need to restart dialogue, there is a group the is strangely absent and silent in the media: the Brigade des Mineurs (juvenile police). The Brigade was created in 1958/9 and replaced the juvenile protection service of 1950 which had, itself, succeeded the specialised corps of Police Assistants that had appeared in the middle of the 30's. The Brigade had initially been intended to prevent juvenile delinquency and it was not until much later that it started to deal with child victims. The silence of historians can be added to this surprising silence of a specialised corps with over 50 years of practical experience. Apart from some works about policewomen written by some contemporary historians of female history, there is currently no analysis of the creation and field experiences of this specific police corps. However, police reviews, specialised social work reviews and numerous reports and brochures from 1950 to 1970 report planned and completed actions in France and neighbouring countries but now seem to have been completely forgotten.

\section{AUTEUR}

\section{MATHIAS GARDET}

Historien, maître de conférences en sciences de l'éducation, Circeft-histoire, université Paris 8 Saint-Denis. 